\title{
Programmable optical transport of particles in knot circuits and networks
}

\author{
José A. Rodrigo*, Mercedes Angulo and Tatiana Alieva ${ }^{1}$ \\ ${ }^{1}$ Universidad Complutense de Madrid, Facultad de Ciencias Físicas, Ciudad Universitaria s/n, Madrid 28040, Spain \\ *Corresponding author: jarmar@fis.ucm.es
}

Compiled August 2, 2018

A freestyle single-beam laser trap allows for multiparticle optical transport along arbitrary open or closed trajectories with independent control of the all-optical confinement and propulsion forces exerted over the particles. Here, exploiting this manipulation tool, we propose and experimentally demonstrate an optical dynamic routing technique to assist multi-particle transport in knot circuits and networks exhibiting multiple crossing paths. This new functionality for optical transport enables the particle circulation in such complex systems handling traffic jams and making possible particle separation/mixing in them. It is important for the development of programmable particle delivery and other automated optical transport operations of interest in colloidal physics, optofluidics, biophysics, etc. (C) 2018 Optical Society of America

OCIS codes: (140.3300) Laser beam shaping; (140.7010) Laser trapping; (170.4520) Optical confinement and manipulation.

http://dx.doi.org/10.1364/ol.XX.XXXXXX

Optical tweezers [1] consisting in point-like laser trap (strongly focused single beam) have been widely adopted as a contactless tool for manipulation of micro/nano-objects in fields such as biophysics, colloidal physics, micro-fabrication, etc. They are often used to manipulate single objects as for example cells and particles. In particular, by moving the position of the focus (the laser trap) the particle can be translated in 3D following a prescribed path. This simple manipulation mechanism can be applied for transport of multiple particles through time-sharing [2-4]. Specifically, several particles may be simultaneously transported if the focused laser beam visits each trap location sufficiently often. Time-sharing has the benefit of being optically simple, however, the number and position of the traps makes challenging the transport of numerous particles, whose control can be a computationally demanding task. An alternative are holographic optical tweezers based on arrays of point-like laser traps created by a computer generated hologram [5]. However, to transport the particles it requires time multiplexing of many holograms that have to be calculated for each motion state, thus resulting in a computationally demanding approach. Therefore, in practice, optical tweezers are mostly applied for manipulation of one or a few particles.
The development of alternative optical manipulation tools suited for multi-particle transport has attracted increased interest in the last decades. For instance, it has been demonstrated that propelling optical forces arising from phase gradients of certain structured trapping beams can be exploited for continuous transport of numerous particles along trajectories defined by the beam's shape [6-12]. A well-known example is the optical vortex ring-like trap able to drive colloidal micro-particles through water along a circular path, making it attractive for micro-pumping applications [6-8]. It has also been found that two or three particles circulating around such a trap undergo periodic collective motion due to their hydrodynamic coupling $[13,14]$. Specifically, these particles can display unexpected dynamical correlations where the path's curvature breaks the symmetry of the two-body hydrodynamic interaction resulting in particle pairing [14]. These facts have attracted the interest of the colloidal physics community in the use of optical transport, which allows creating and studying micro-systems of colloidal particles undergoing in complex collective motions [15-17].

The recently introduced freestyle laser trap [11, 12] allows for simultaneous optical confinement and transport of numerous particles along arbitrary 3D curves (trajectories). The freestyle laser trap is a type of single-beam trap whose high intensity and phase gradients prescribed along the curve are responsible for the confinement and propelling optical forces exerted over the particles, respectively. Furthermore, these optical forces and the shape of the particle trajectory are independently controlled. Thus, the freestyle laser trap is promising for the development of optical manipulation tools. Moreover, it can also be easily implemented by using only a single computer generated hologram. Its application for multi-particle optical transport along 2D and 3D open/closed trajectories has been experimentally demonstrated on the example of dielectric micro-particles (silica spheres of $1 \mu \mathrm{m}$ ) [11, 12], plasmonic nano-particles (gold and silver, size of $100 \mathrm{~nm}$ ) [18], and even biological cells (rod-like bacteria $1 \mu \mathrm{m}$ wide and $4 \mu \mathrm{m}$ long) [19].

In practice, multi-particle transport operations such as programmable particle delivery require optical manipulation tools as versatile as possible. For example, the flexible design of the freestyle laser trap enables easily-programmable shape transformation of the particle trajectory (of the curved laser trap) providing obstacle avoidance necessary in particle delivery [18, 20], etc. Other multi-particle transport problems, however, are more challenging. This is the case of optical transport of numerous 
particles in knot circuits or networks exhibiting multiple crossing points where without a proper routing control the particles can collide each other, thereby interrupting the particle transport or delivery. In this Letter, we propose a technique based on the freestyle laser trap to handle this optical transport problem. It consists in the temporal reconfiguration of the curved laser trap to achieve dynamic routing of the trapped particles, a functionality required for programmable control of the particle transportation. Another advantage of dynamic routing is that it assists for programmable transport enabling separation and re-distribution of the particles across the knot circuit and network. The performance of the proposed technique is experimentally demonstrated on the example of colloidal dielectric micro-particles (silica spheres of $1 \mu \mathrm{m}$ dispersed in de-ionized water) optically transported along knot circuits of different complexity.

Let us first summarize the fundamentals of the freestyle laser trap considered in this work for multi-particle transport along $2 \mathrm{D}$ trajectories. It is well-known that the high-intensity gradients of the beam are responsible for the optical confinement (trapping) forces of the familiar optical tweezers [1]. While, the phase gradients can redirect part of the radiation pressure of a structured trapping beam yielding a transverse optical force suited for the particle transportation [8]. The freestyle laser trap $[11,12]$ exploits both types of forces for all-optical confinement and transportation of particles along arbitrary curves. To create a freestyle laser trap with intensity and phase distributions prescribed along a parametric 2D curve, given in polar coordinates $\mathbf{c}(t)=(R(t) \cos t, R(t) \sin t)$, it is required the focusing of the polymorphic beam $[11,12]$

$$
E\left(\mathbf{r}_{0}\right)=\int_{0}^{T} g(t) \exp \left[\frac{\mathrm{i} k}{\mathrm{f}} \mathbf{r}_{0} \mathbf{c}(t)\right] \mathrm{d} t
$$

where $\mathbf{r}_{0}=\left(x_{0}, y_{0}\right)$ are spatial coordinates in the input plane of the focusing lens whose focal length is $f$. The parameter $T$ stands for the maximum value of the azimuth angle $t$, where $k=2 \pi / \lambda$ with $\lambda$ being the light wavelength. The function $g(t)=|g(t)| \exp [\mathrm{i} \Psi(t)]$ plays a crucial role in the design of the curved laser trap: Its modulus controls the intensity distribution along the curve while its phase is responsible for the particle propulsion along it. Specifically, the intensity is uniform along the curve when $|g(t)| \propto\left|\mathbf{c}^{\prime}(t)\right|$ whereas the phase is independently prescribed by $\Psi(t)=2 \pi l S(t) / S(T)$ along the curve, with $S(t)$ being an arbitrary real function. For instance, the phase distribution can be uniform along a curve $\mathbf{c}(t)$ - contained in the focal plane, with coordinates $\mathbf{r}=(x, y)-$ by using $S(t)=\int_{0}^{t}\left|\mathbf{c}^{\prime}(\tau)\right| \mathrm{d} \tau$, or be non-uniform if the particles need to be speed up or slow down at certain points along the trajectory $[11,12]$. Note the parameter $l$ defines the phase accumulation along the entire curve, whose length is $L=\int_{0}^{T}\left|\mathbf{c}^{\prime}(\tau)\right| \mathrm{d} \tau$. The direction of the phase gradient coincides with the curve tangent $\mathbf{v}=\mathrm{d} \mathbf{c} /|\mathrm{d} \mathbf{c}|$ and then $\nabla \Psi=\mathbf{v} \cdot \mathrm{d} \Psi / \mathrm{d} \mathbf{c}$, where $\mathrm{d} \Psi / \mathrm{d} \mathbf{c}=2 \pi l S^{\prime}(t) /\left[S(T)\left|\mathbf{c}^{\prime}(t)\right|\right]$. The particles experience a propelling force $\mathbf{F}_{\mathbf{v}} \propto \mathbf{j}(\mathbf{r})$ proportional to the optical current [21] defined as $\mathbf{j}(\mathbf{r})=I(\mathbf{r}) \nabla \Psi(\mathbf{r})$, where $I(\mathbf{r})$ and $\Psi(\mathbf{r})$ are the intensity and phase distributions of the beam with $\nabla$ being the gradient in the transverse plane (e.g., trapping plane), correspondingly. Here, we consider 2D laser curves with uniform intensity $I$ and phase distribution $\Psi(t)$ for which the optical current is constant: $\mathbf{j}=I 2 \pi l L^{-1} \mathbf{v}$. The polymorphic beam Eq. (1) is encoded as a hologram addressed into a spatial light modulator (SLM, Holoeye PLUTO, pixel size of $8 \mu \mathrm{m}$ ) [11, 12].
While, the microscope's objective lens focuses it in form of curve $25 \mu \mathrm{m}$ deep within the sample to prevent from perturbations due to proximal wall effects.

To introduce the fundamentals of dynamic routing, let us first consider the particle transport problem along a 2D knot circuit as the eight-shaped curve $c$ displayed in Fig. 1(a). We have applied a method for representing the curve $\mathbf{c}$ as a piecewisedefined smooth curve $\mathbf{c}=\left\{\mathbf{b}_{1}, \ldots, \mathbf{b}_{m}\right\}$ by using a set of $m$ parametric Bézier splines $\mathbf{b}$ (cubic polynomials) linked each other [22], which has been recently exploited for shape control of 3D curved laser traps [20]. This method is particularly useful for both creating arbitrary knot circuits and re-configuring their crossing paths. In our case, the eight-shaped knot circuit (of length $L=36.5 \mu \mathrm{m}$ ) has been created by using $m=18$ Bézier splines, corresponding with the colored segments indicated in Fig. 1(a). This circuit has one crossing of paths that requires particle traffic control similar to a railroad switch. To achieve such a traffic control, the curve $\mathrm{c}$ has to be temporally reconfigured enabling the particle routing. For example, to let the particles crossing along the path 1-2 the curve segments 3 and 4 have to be removed, as indicated in the first routing configuration shown in Fig. 1(a). We underline that the removed curve segments should leave a hollow a little bit bigger than the diameter of the particles to ensure their crossing. Once the particles reach the end of the curve, labeled by $w$ in Fig. 1(a), the curve $\mathrm{c}$ is again reconfigured to let the particles crossing now along the path $3-4$, just by removing the segments 1 and 2 . Note that for a static routing configuration the particles stop at the curve's end $w$ waiting for the next crossing state. Therefore, the dynamic routing process involves controlled updating of the crossing states similar to switch rails. This example illustrates how the curve c can be easily reconfigured to achieve dynamic routing with a freestyle laser trap that enables the optical transport of particles in knot circuits.

For this knot circuit there exists four different configurations or trap states $\mathbf{c}_{1, \ldots, 4}$, see Fig. 1(b), that can be temporally activated to create a dynamic routing process allowing for optical transport along the whole circuit including the capability of particle separation. Specifically, in Fig. 1(b), it has been displayed for each routing configuration the intensity and phase distribution (for $l=30$ ) of the corresponding curved laser trap along with the normalized modulus of the calculated propelling force $\mathbf{F}_{\mathbf{v}} \propto \mathbf{j}(\mathbf{c})$. It is expected that in the first trap state $c_{1}$ (where the splines 2 and 3 have been removed) the particles can be transported along the bigger circle in which they are confined performing a continuous clockwise rotation. When the second trap state $c_{2}$ is activated some (or all) of these particles can be optically transported toward the small circle comprising the knot circuit, where they can be retained performing a continuous anti-clockwise rotation when the third trap state is activated. Finally, the particles retained in the small circle can be transferred back to the bigger one just by activating the fourth trap state $c_{4}$ (splines 1 and 2 have been removed). The described dynamic routing process can be continuously repeated if needed. This optical transportation process, comprising controlled routing and separation of the particles in the knot circuit, has been experimentally verified by using multiple particles as observed in Fig. 1(c) and Visualization 1. All the particles have been tracked [23] revealing a trajectory that coincides with the programmed knot circuit as expected, see Fig. 1(d) and Visualization 1. From the particle tracking it has been found that the expected speed of the transported particles is $6-10 \mu \mathrm{m} / \mathrm{s}$ along the circuit. The time-lapse image displayed in Fig. 1(e), created by combining all the frames 
(a) Knot circuit
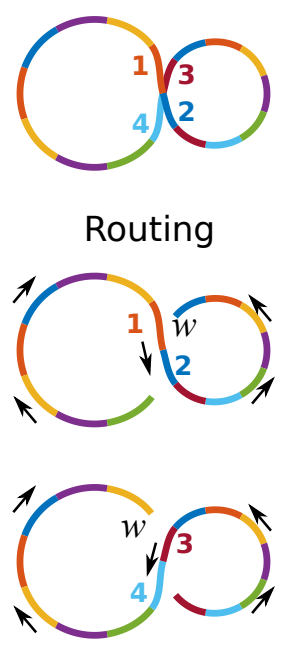

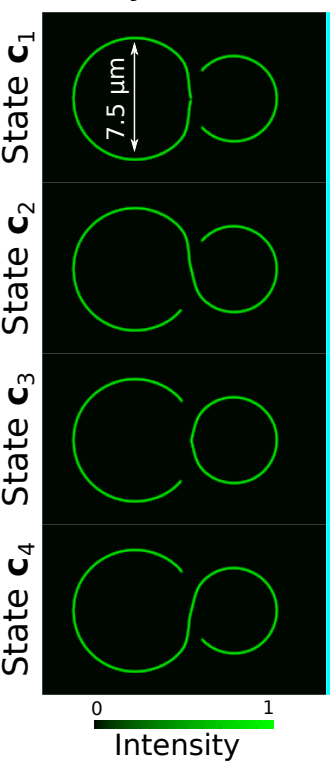

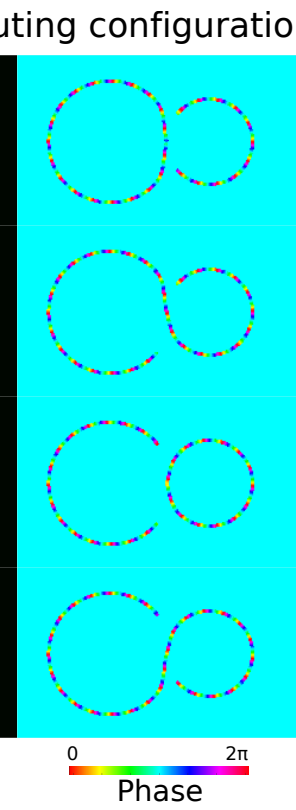

(c)

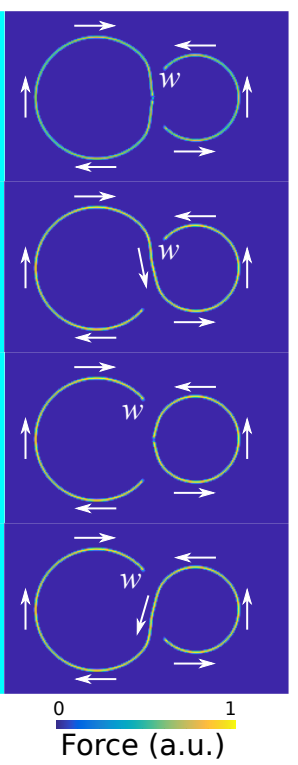

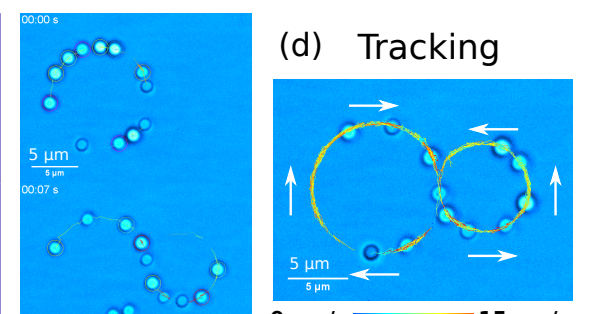

$0 \mu \mathrm{m} / \mathrm{s} \quad 15 \mu \mathrm{m} / \mathrm{s}$

\section{(e) Time lapse}

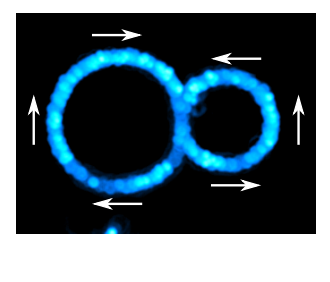

(f)
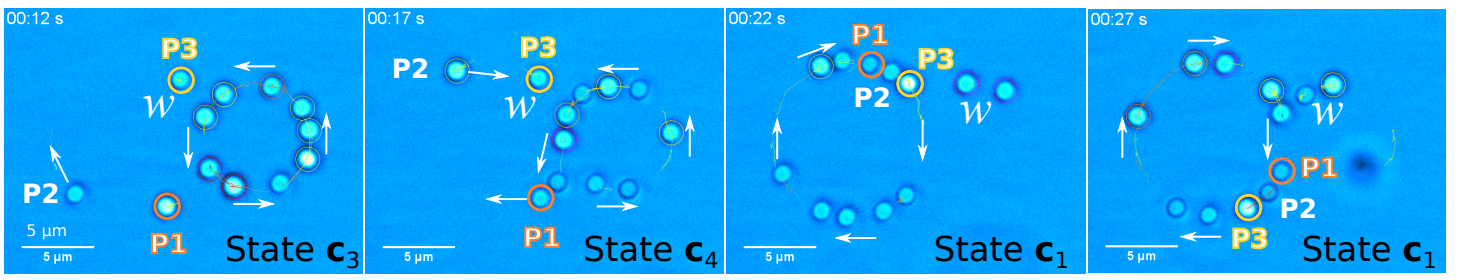

Fig. 1. (a) Knot circuit in form of eight-shaped curve c (constructed with $m=18$ splines) and an example of routing configurations. (b) The intensity and phase distributions of the numerically calculated curved laser traps, along with the propelling force $\left|\mathbf{F}_{\mathbf{v}}\right|$ defined along the curve, have been displayed for the considered routing configurations (trap states $\mathrm{c}_{1, \ldots, 4}$ ). (c)-(f) Experimental results of the optical transport of multiple particles along the circuit, see Visualization 1.

of Visualization 1, also confirms that the particles have been properly confined and transported according the shape of the knot circuit, Fig. 1(a).

This experiment proves the success of dynamic routing for programmable multi-particle transport in knot circuits including the ability of controlled separation of the particles: They can be retained in a part of the circuit for a given time and then be transferred to a different part as required. It is observed (see Visualization 1) several particles crossing the intersecting path while other stand in a queue, for example, as shown in Fig. 1(f). Specifically, Fig. 1(f) displays the sequence corresponding to the transition from the trap state $c_{3}$ (starting at $12 \mathrm{~s}$ in Visualization 1) to the $c_{1}$. Nine particles are retained in the small circle (performing anit-clockwise rotation) of the state $\mathbf{c}_{3}$ while only three particles (labeled as P1, P2 and P3 ) are retained in its c-shaped curve. The particle $\mathrm{P} 1$ and $\mathrm{P} 3$ remain at the ends of the c-shaped curve of $c_{3}$ while the particle P2 is optically transported along it following the clockwise direction. Once the particle $\mathrm{P} 2$ reaches the position of $\mathrm{P} 3$ they remain in queue until the activation of the trap state $c_{1}$ (at time $22 \mathrm{~s}$ ) that allows them crossing the updated path segment and thus be optically transported along the rest of the circuit. Note that the queue particles in the circuit region $w$ wait for crossing the path as indicated in Fig. 1(f).

We underline that the optical transport of the particles along the eight-shaped circuit is also possible by only using the trap states $\mathbf{c}_{2}$ and $\mathbf{c}_{4}$, but in this case without the practical functionality of controlled separation of the particles previously described.
Another relevant fact is that the value of the switching time between consecutive trap states can be varied during the experiment depending on the required particle routing and separation tasks. Here, as an example, we have used a switching time as short as $5 \mathrm{~s}$. For switching times below $200 \mathrm{~ms}$ - the minimum time that our SLM can display two consecutive holograms is $30 \mathrm{~ms}$ - we have found that the particle transport is interrupted at the crossing paths. This is because the particles feel a fast timeshared trap resulting in the knot circuit with fixed crossing paths, see Fig. 1(a), that obstructs the particle transport as previously mentioned.

The proposed dynamic routing process also allows for multiparticle transport between or across several circuits in form of networks comprising multiple crossing paths. An example of such a network is the trefoil knot displayed in Fig. 2(a) that has three crossing paths. In this case we have considered two routing configurations, corresponding to the trap states $\mathbf{c}_{1}$ and $c_{2}$ displayed in Fig. 2(b), that have been activated each $5 \mathrm{~s}$ (switching time) to achieve programmable multi-particle transport as observed in Visualization 2. The length of this circuit is $L=38.3 \mu \mathrm{m}$ and the calculated intensity, phase $(l=30)$ as well as propelling force $\mathbf{F}_{\mathbf{v}}$ for each trap state are shown in Fig. 2(b). The tracking of the particles and the time-lapse image confirm that indeed the trajectory of the particles fits the shape of the network (trefoil knot) as shown in Fig. 2(c). Moreover, in Visualization 2 it is observed the queue of particles (at the curve's ends $w$ ) that yield to other particles crossing the intersecting path each 5 seconds, according to the switching time of the trap 
(a) Knot circuit
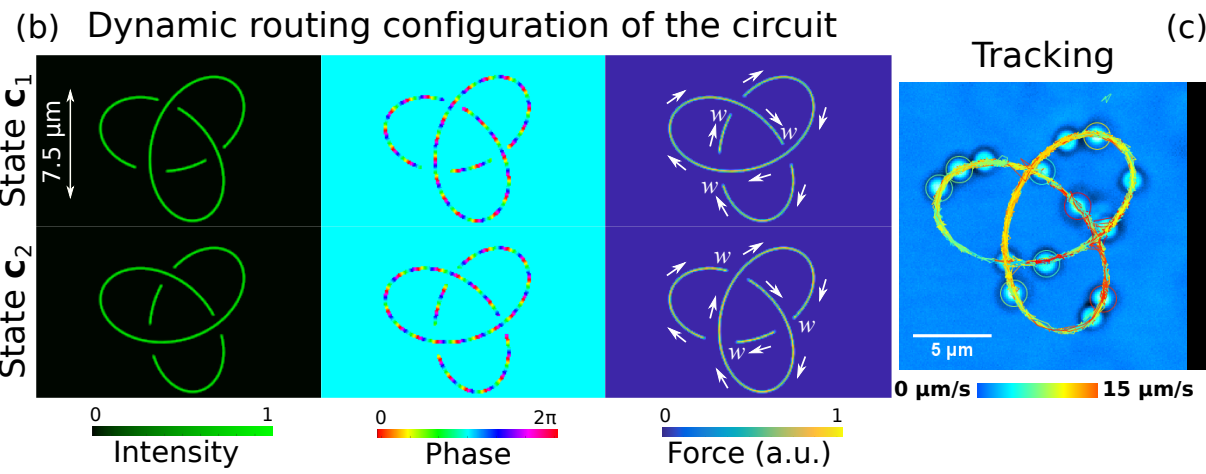

(c) Time lapse
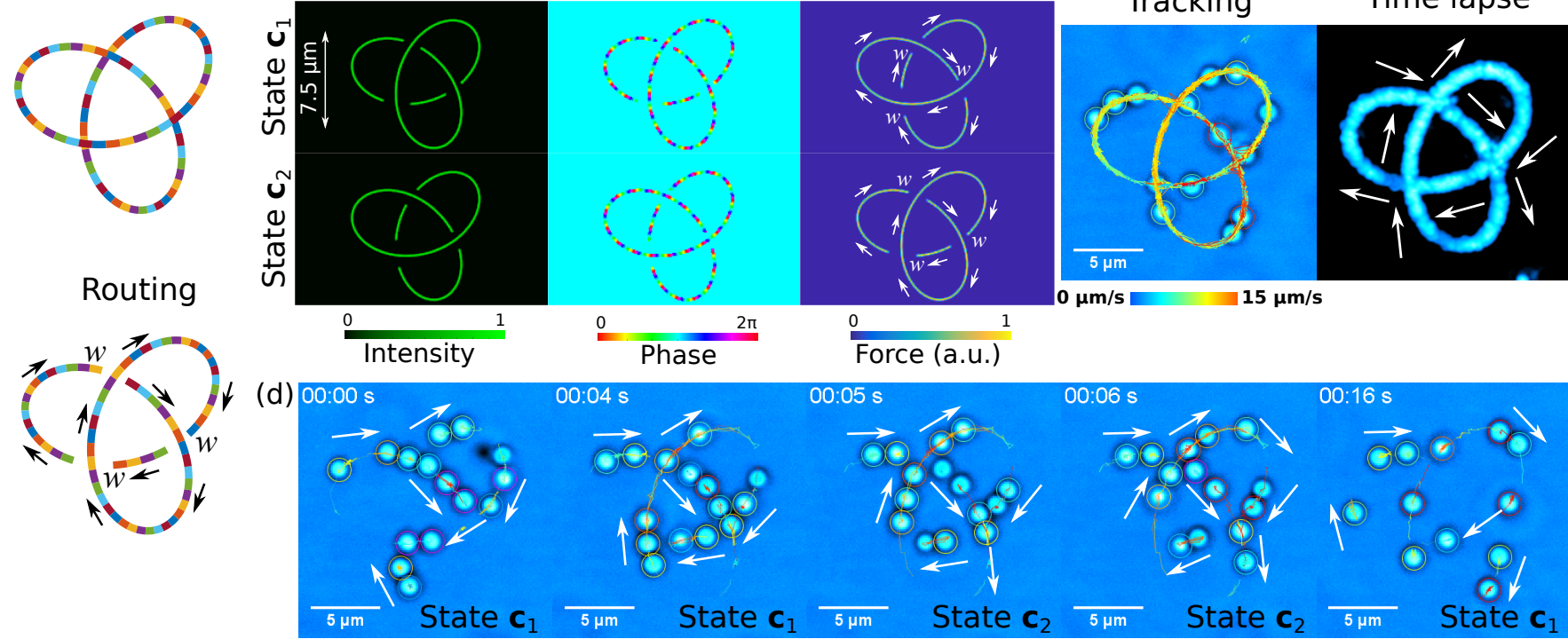

Fig. 2. (a) Trefoil knot circuit c (constructed with $m=79$ splines) and an example of routing configuration. (b) The intensity and phase distributions of the numerically calculated curved laser traps, along with the propelling force $\left|\mathbf{F}_{\mathbf{v}}\right|$ defined along the curve, have been displayed for the considered routing configurations (trap states $\mathrm{c}_{1,2}$ ). (c)-(d) Experimental results of the optical transport of multiple particles along the circuit, see Visualization 2.

states $\mathbf{c}_{1}$ and $\mathbf{c}_{2}$, as expected. The sequence of images displayed in Fig. 2(d), extracted from Visualization 2, shows some queue particles waiting to pass through an intersection of paths while the others are optically transported. In Visualization 2 it is also observed that a waiting particle can eventually escape through the path's edge (at the curve's end $w$ indicated in Fig. 2(b)) due to the collisions with rear particles. Such rear particles can push the waiting particle overcoming the confinement force that this particle experiences at the curve's end $w$.

The latter example shows that the separation and routing of the particles can be more sophisticated when all the crossing paths are reconfigured as routers of a network. For instance, a set of trailing particles can be separated by routing them in different paths with the option of recombining them back or mixing them with other particles confined in the network (e.g., trefoil knot). The experimental results illustrate the performance of optical dynamic routing for multi-particle transport operations such as programmable particle delivery including separation and re-distribution of the particles across a knot circuit or network. We envision that the combined use of freestyle laser traps $[11,20]$ and the proposed dynamic routing technique can be exploited for the creation of complex networks enabling transport of colloidal particles, study of their interactions, and providing sophisticated operations of interest in colloidal physics, optofluidics, biophysics, etc.

\section{FUNDING}

The Ministerio de Economía y Competitividad is acknowledged for funding the project TEC2014-57394-P.

\section{REFERENCES}

1. A. Ashkin, Optical Trapping and Manipulation of Neutral Particles Using Lasers (World Scientific, 2006).

2. K. Sasaki, M. Koshioka, H. Misawa, N. Kitamura, and H. Masuhara, Opt. Lett. 16, 1463 (1991).
3. R. Nambiar and J.-C. Meiners, Opt. Lett. 27, 836 (2002).

4. M. Capitanio, R. Cicchi, and F. S. Pavone, Opt. Lasers Eng. 45, 450 (2007).

5. S. Bianchi and R. D. Leonardo, Comput. Phys. Commun. 181, 1444 (2010).

6. H. Rubinsztein-Dunlop, T. a. Nieminen, M. E. J. Friese, and N. R. Heckenberg, Adv. Quantum Chem. 30, 469 (1998).

7. K. Ladavac and D. Grier, Opt. Express 12, 1144 (2004).

8. Y. Y. Roichman, B. Sun, Y. Y. Roichman, J. Amato-Grill, and D. G. Grier, Phys. Rev. Lett. 100, 8 (2008).

9. S.-h. S. Lee, Y. Roichman, and D. D. G. Grier, Opt. Express 18, 1974 (2010).

10. E. R. Shanblatt and D. G. Grier, Opt. Express 19, 5833 (2011).

11. J. A. Rodrigo and T. Alieva, Optica 2, 812 (2015).

12. J. A. Rodrigo and T. Alieva, Sci. Rep. 6, 35341 (2016).

13. Y. Roichman, D. G. Grier, and G. Zaslavsky, Phys. Rev. E - Stat. Nonlinear, Soft Matter Phys. 75, 1 (2007).

14. Y. Sokolov, D. Frydel, D. G. Grier, H. Diamant, and Y. Roichman, Phys. Rev. Lett. 107, 1 (2011).

15. Y. Sassa, S. Shibata, Y. Iwashita, and Y. Kimura, Phys. Rev. E - Stat. Nonlinear, Soft Matter Phys. 85, 6 (2012).

16. H. Nagar and Y. Roichman, Phys. Rev. E - Stat. Nonlinear, Soft Matter Phys. 90, 5 (2014).

17. S. Okubo, S. Shibata, Y. S. Kawamura, M. Ichikawa, and Y. Kimura, Phys. Rev. E 92, 032303 (2015).

18. J. A. Rodrigo and T. Alieva, Sci. Rep. 6, 33729 (2016).

19. J. A. Rodrigo, J. M. Soto, and T. Alieva, Biomed. Opt. Express 8, 5507 (2017).

20. J. A. Rodrigo, M. Angulo, and T. Alieva, Opt. Express 26, 18608 (2018).

21. M. V. Berry, J. Opt. A Pure Appl. Opt. 11, 094001 (2009).

22. J. D. Hobby, Discret. \& Comput. Geom. 1, 123 (1986).

23. J.-Y. Tinevez, N. Perry, J. Schindelin, G. M. Hoopes, G. D. Reynolds, E. Laplantine, S. Y. Bednarek, S. L. Shorte, and K. W. Eliceiri, Methods 115, 80 (2017). 


\section{REFERENCES}

1. A. Ashkin, Optical Trapping and Manipulation of Neutral Particles Using Lasers (World Scientific, 2006).

2. K. Sasaki, M. Koshioka, H. Misawa, N. Kitamura, and H. Masuhara, "Pattern formation and flow control of fine particles by laser-scanning micromanipulation," Opt. Lett. 16, 1463-1465 (1991).

3. R. Nambiar and J.-C. Meiners, "Fast position measurements with scanning line optical tweezers," Opt. Lett. 27, 836-838 (2002).

4. M. Capitanio, R. Cicchi, and F. S. Pavone, "Continuous and time-shared multiple optical tweezers for the study of single motor proteins," Optics and Lasers in Engineering 45, 450 - 457 (2007).

5. S. Bianchi and R. D. Leonardo, "Real-time optical micro-manipulation using optimized holograms generated on the GPU," Comput. Phys. Commun. 181, 1444-1448 (2010).

6. H. Rubinsztein-Dunlop, T. a. Nieminen, M. E. J. Friese, and N. R. Heckenberg, "Optical Trapping of Absorbing Particles," Adv. Quantum Chem. 30, 469-492 (1998).

7. K. Ladavac and D. Grier, "Microoptomechanical pumps assembled and driven by holographic optical vortex arrays." Opt. Express 12, 1144-9 (2004).

8. Y. Y. Roichman, B. Sun, Y. Y. Roichman, J. Amato-Grill, and D. G. Grier, "Optical Forces Arising from Phase Gradients," Phys. Rev. Lett. 100, 8-11 (2008).

9. S.-h. S. Lee, Y. Roichman, and D. D. G. Grier, "Optical solenoid beams," Opt. Express 18, 1974-1977 (2010).

10. E. R. Shanblatt and D. G. Grier, "Extended and knotted optical traps in three dimensions." Opt. Express 19, 5833-5838 (2011).

11. J. A. Rodrigo and T. Alieva, "Freestyle $3 D$ laser traps: tools for studying light-driven particle dynamics and beyond," Optica 2, 812-815 (2015).

12. J. A. Rodrigo and T. Alieva, "Polymorphic beams and Nature inspired circuits for optical current," Sci. Rep. 6, 35341 (2016).

13. Y. Roichman, D. G. Grier, and G. Zaslavsky, "Anomalous collective dynamics in optically driven colloidal rings," Phys. Rev. E - Stat. Nonlinear, Soft Matter Phys. 75, 1-4 (2007).

14. Y. Sokolov, D. Frydel, D. G. Grier, H. Diamant, and Y. Roichman, "Hydrodynamic pair attractions between driven colloidal particles," Phys. Rev. Lett. 107, 1-5 (2011).

15. Y. Sassa, S. Shibata, Y. Iwashita, and Y. Kimura, "Hydrodynamically induced rhythmic motion of optically driven colloidal particles on a ring," Phys. Rev. E - Stat. Nonlinear, Soft Matter Phys. 85, 6-10 (2012).

16. H. Nagar and Y. Roichman, "Collective excitations of hydrodynamically coupled driven colloidal particles," Phys. Rev. E - Stat. Nonlinear, Soft Matter Phys. 90, 5 (2014).

17. S. Okubo, S. Shibata, Y. S. Kawamura, M. Ichikawa, and Y. Kimura, "Dynamic clustering of driven colloidal particles on a circular path," Phys. Rev. E 92, 032303 (2015).

18. J. A. Rodrigo and T. Alieva, "Light-driven transport of plasmonic nanoparticles on demand," Sci. Rep. 6, 33729 (2016).

19. J. A. Rodrigo, J. M. Soto, and T. Alieva, "Fast label-free microscopy technique for $3 d$ dynamic quantitative imaging of living cells," Biomed. Opt. Express 8, 5507-5517 (2017).

20. J. A. Rodrigo, M. Angulo, and T. Alieva, "Dynamic morphing of 3D curved laser traps for all-optical manipulation of particles," Opt. Express 26, 18608-18620 (2018).

21. M. V. Berry, "Optical currents," J. Opt. A Pure Appl. Opt. 11, 094001 (2009).

22. J. D. Hobby, "Smooth, easy to compute interpolating splines," Discrete \& Computational Geometry 1, 123-140 (1986).

23. J.-Y. Tinevez, N. Perry, J. Schindelin, G. M. Hoopes, G. D. Reynolds, E. Laplantine, S. Y. Bednarek, S. L. Shorte, and K. W. Eliceiri, "Trackmate: An open and extensible platform for single-particle tracking," Methods 115, $80-90$ (2017). 